\title{
Meeting physical activity recommendations is associated with health- related quality of life in women before and after Roux-en-Y gastric bypass surgery
}

\author{
Fanny Sellberg $^{1}$ (D) $\cdot$ Sofie Possmark ${ }^{1} \cdot$ Mikaela Willmer $^{2} \cdot$ Per Tynelius $^{1,3} \cdot$ Margareta Persson $^{4} \cdot$ Daniel Berglind $^{1}$
}

Accepted: 23 January 2019 / Published online: 5 February 2019

(c) The Author(s) 2019

\begin{abstract}
Purpose Meeting physical activity (PA) recommendations is positively associated with health-related quality of life (HRQoL), but it is still unclear whether PA (specifically objectively measured) is associated with HRQoL in bariatric surgery candidates, both before and after surgery. Thus, the aim of this study was to examine the cross-sectional association between meeting objectively measured PA recommendations and HRQoL before and after Roux-en-Y gastric bypass (RYGB) surgery. Methods Sixty-six women undergoing RYGB with pre-surgery and 62 women with post-surgery valid PA and HRQoL data were included from the control group of a RCT study aiming at improving HRQoL and PA post-RYGB surgery. Measures before and 12 months after RYGB included the Short Form Health Survey (SF-36) and objectively measured PA, sedentary time (ST), and step counts with GT3X+ accelerometers. Multiple linear regression models were used to analyze the associations between PA and HRQoL.

Results Participants who engaged in more than 150 min of moderate to vigorous PA (MVPA)/week (PA recommendations) had considerably higher SF-36 scores (HRQoL) than those who did not, both pre and 12-month post-surgery, with greatest difference in the subscale bodily pain, $15.5(p=0.021)$ higher score (higher scores means less pain) pre-surgery and a 19.7 $(p=0.004)$ higher score post-surgery. Higher LPA and step counts and lower ST also showed positive associations in some of the subscales of SF-36.

Conclusions Meeting the PA recommendations and overall engaging in more PA was associated with higher HRQoL, pre-, and post-RYGB surgery, highlighting the importance of PA both pre- and post-surgery.
\end{abstract}

Keywords Health-related quality of life $\cdot$ Physical activity $\cdot$ Bariatric surgery $\cdot$ Gastric bypass $\cdot$ Sedentary time $\cdot$ Step counts

Electronic supplementary material The online version of this article (https://doi.org/10.1007/s11136-019-02120-0) contains supplementary material, which is available to authorized users.

Fanny Sellberg

fanny.sellberg@ki.se

Sofie Possmark

sofie.possmark@ki.se

Mikaela Willmer

mikaela.willmer@hig.se

Per Tynelius

per.tynelius@ki.se

Margareta Persson

margareta.persson@umu.se

Daniel Berglind

daniel.berglind@ki.se
1 Department of Public Health Sciences (PHS), Karolinska Institutet, K9, Social Medicin, 17177 Stockholm, Sweden

2 Department of Health and Caring Sciences, University of Gävle, 80176 Gävle, Sweden

3 Centre for Epidemiology and Community Medicine, Stockholm County Council, Box 45436, 10431 Stockholm, Sweden

4 Department of Nursing, Umeå University, 90187 Umeå, Sweden 


\section{Introduction}

Meeting the physical activity (PA) recommendations of at least $150 \mathrm{~min}$ of moderate to vigorous PA (MVPA) per week is associated with a wide range of positive health outcomes across all weight ranges, including reduced risk of heart disease, type 2 diabetes, some cancers, and improved mental health [1, 2]. Furthermore, meeting the PA recommendations and higher duration, intensity, and bout length of PA are positively associated with healthrelated quality of life (HRQoL) [3-5]; a multidimensional measure of physical, mental, functional, and social wellbeing, in the general population [6]. Less is known about sedentary time (ST) and PA associations with HRQoL in individuals suffering from obesity $[7,8]$ and few studies have used objectively measured PA and ST [9].

Individuals suffering from obesity often show lower levels of HRQoL compared to the general normal weight population [10]. Weight loss has been associated with increases in HRQoL [11], especially rapid weight loss induced by bariatric surgery [12-14], where Roux-en-Y gastric bypass (RYGB) is the most commonly performed bariatric surgery procedure in Sweden [15]. Greatest improvements of HRQoL, induced by weight loss, are often seen on the physical part of the measurement scale, probably caused by the reduced physical complaints after losing weight, but the mental part has also been shown to improve $[12,13]$.

Peak improvements in HRQoL after bariatric surgery are typically observed during the first years of follow-up, followed by a small decline that usually stabilizes approximately 5 years postoperatively $[16,17]$. Although HRQoL improves substantially after bariatric surgery, PA usually does not increase (despite great weight loss) [18-20], except when self-reported PA is used as an outcome measure [20]. The discrepancy between self-reported and objectively measured PA in this patient group is large, and tends to increase post-RYGB [21]. Consequently, objectively measured PA is the preferred method for assessing actual levels of PA in this patient category.

Interventions aiming to increase PA pre-bariatric surgery have been shown to also improve HRQoL pre-surgery [22], but not post-surgery [23]. However, it is still unclear whether objectively measured PA is associated with HRQoL before and after bariatric surgery in candidates receiving regular care (in a non-intervention setting). Thus far, 10-year follow-up data from the Swedish Obese Subjects (SOS) study have shown that HRQoL is improved and associated with weight loss after bariatric surgery. However, the self-reported data on PA, with its inherent bias [24], limit any interpretation on associations between PA and HRQoL before and after RYGB.
The aim of this study was to examine the association between meeting physical activity recommendations, light PA (LPA), ST and step counts (objectively measured), and HRQoL before and 12 months after RYGB surgery. A secondary aim was to explore the association between pattern and intensity of PA and HRQoL changes pre- and 12-month post-RYGB.

\section{Materials and methods}

For the current study, we used the control group of an ongoing randomized controlled intervention, to study the association between PA and HRQoL. The study, named WELL-RYGB, has been described in detail in a previously published protocol paper [25]. In short, the WELLRYGB is a randomized controlled trial examining the effects of a dissonance-based post-RYGB intervention, on HRQoL and PA at 12- and 24-month follow-up. The study was approved by the regional ethics committee of Stockholm (Dnr:2013/1847-31/2). The trial has also been registered at http://www.isrctn.org with identification number ISRCTN16417174 and all participants have given oral and written consent to participate.

\section{Participants}

Women were recruited from waiting lists for RYGB surgery from five different hospitals in Sweden (Örebro University Hospital, Akademiska Hospital, Ersta Hospital, St. Görans Hospital, and Danderyds Hospital). Inclusion criteria were being eligible for RYGB surgery [body mass index $(\mathrm{BMI})>35$ with complications from the obesity or BMI $>40$, conducted several serious attempts to lose weight with other methods, and usually over 18 years old] and enough Swedish language skills to be a part of an intervention in Swedish and answer questionnaires in Swedish. Interested patients were sent informed consent forms, a questionnaire measuring HRQoL and demographic characteristics, and an accelerometer before surgery. Participants were included if they returned the informed consent. The same questionnaires and an accelerometer were sent 12 months after surgery, yielding two measure points: baseline (approximately 1 month before RYGB) and 12-month follow-up. We only included the control group in this substudy since the intervention aimed at improving HRQoL and PA and may therefore, if included in the current study, bias the results. Additionally, there were only women included in the original intervention study because of power concerns. The intervention might affect men and women differently, therefore creating the need for stratification, and it was not possible to collect the double number of participants in order to obtain enough power, especially as approximately $77 \%$ of 
all Swedish RYGB patients are women [15]. All participants had RYGB surgery between April 2015 and June 2017. In the current sub-study, we included 66 women with pre-surgery and 62 women with 12-month post-surgery valid PA and HRQoL data, only 39 women had valid accelerometer data for both measure points.

\section{HRQoL}

HRQoL was measured using the Short Form (36) Health Survey (SF-36). The SF-36 is divided into eight domains: vitality (VT), physical functioning (PF), bodily pain (BP), general health perceptions $(\mathrm{GH})$, physical role functioning (RP), emotional role functioning (RE), social role functioning (SF), and mental health (MH), and can be summarized into two summary scores: physical summary score (PCS) and mental summary score (MCS). Scoring ranges from 0 to 100 with higher scores indicating better quality of life. The instrument performs well in a general population [26] and is commonly used in obese populations [11]. SF-36 was scored using the Quality Metric scoring Software 8.6.12. pptx and the "maximum data recovery" setting was used for missing values.

\section{Physical activity}

PA, LPA, step counts, and ST were measured objectively with an accelerometer (ActiGraph GT3X+), worn on the right hip. Participants were asked to wear the accelerometer for all waking hours for seven consecutive days. We used vector magnitude activity counts $\left(\mathrm{V}_{\mathrm{m}}\right)$, calculated as the square root of the sum of the counts on all three axes, recorded in 10-s epochs and then converted into counts per minutes (cpm). Measurements were accepted as valid if participants had worn the accelerometer for at least $10 \mathrm{~h}$ per day during at least 3 days. The number of participants with valid measure at one of the two measure points is reported in Table 2. Wear time, MVPA, and classification of bouts were computed using ActiLife v.6.13.3. For wear time, we used an algorithm by Choi et al. [27]. If there were no counts for 60 consecutive minutes or more, with a maximum break of two minutes with non-zero counts, it was classified as non-wear time [28] and consequently removed from analyses. MVPA minutes were calculated as minutes per day in total and also in 10 min bouts. ST was defined as any minute showing $<100 \mathrm{cpm}$, light physical activity (LPA) was defined as 100-3207 cpm, and MVPA as $3208 \mathrm{cpm}$ or more [29].

\section{Other variables}

Weight and height were objectively measured at the hospitals, in a standardized manner, pre- and 12-month postsurgery and obtained from medical records. Self-reported questionnaire data at baseline and at 1-year follow-up were used to assess current occupation (categorized as working or not working) and level of education (categorized as university level or lower). Long-term sickness was defined by a question asking if participants had a chronic disease, difficulty after an accident, reduced physical function, or long-term health condition. If the respondent answered yes, the following question was asked: does this condition result in reduced work capacity or limit your regular occupation? With response options (1) "not at all," (2) "yes, to some degree," and (3) "yes, to a high degree." BMI was calculated as weight $(\mathrm{kg}) /$ height $^{2}\left(\mathrm{~m}^{2}\right)$.

\section{Statistical analysis}

Data in table one and two are presented as means and standard deviation (SD) or in numbers and percentages. Twotailed $T$ tests were used to test for differences in BMI and PA over time. Multiple regression models were used to analyze the associations between LPA and HRQoL, step counts and HRQoL, ST and HRQoL, and between meeting the MVPA recommendations and HRQoL, with MVPA categorized into two groups, more or less than $150 \mathrm{~min}$ of MVPA/week. A similar approach was used for MVPA performed in 10-min bouts since the recommendation for PA is at least $150 \mathrm{~min}$ of MVPA performed in 10-min bouts (or longer) per week [30]. However, we also analyzed MVPA in non-bouts, since MVPA independent of how it is accumulated, is associated with numerous health benefits [31]. BMI, percent weight loss, and age were not significant confounders and were therefore not included in the adjusted models. Education, occupation, and long-term sickness showed significant confounding effects in several models and were therefore included in all adjusted analyses of MVPA recommendations and HRQoL as well as wear time. For the association between LPA, step counts, ST, and HRQoL, only occupation and long-term sickness were found to be significant confounders and therefore included in the adjusted models, as well as wear time. The majority of the participants only had PA measured at one time-point and there could be systematic differences between those with PA measured at one or two time points. Therefore, sensitivity analyses were performed for the group of participants who had valid PA measures both pre- and post-surgery $(n=39)$ (Supplementary Tables 1 and 2). All statistical analyses were performed with STATA.15.1 (StataCorp).

\section{Results}

Out of the 103 included participants in the study, 90 women had valid accelerometer data for at least one of the measurements, before or 12 months after RYGB. Sixty-six of those 
had valid accelerometer data and HRQoL data pre-surgery, whereas 62 had valid data on the same measures at 12-month post-RYGB. Thirty-nine women had valid data at both measure points. Characteristics of participants pre- and 12-month post-RYGB are presented in Table 1. Participants with both valid accelerometer measurement had overall slightly higher SF-36 scores pre-surgery, suffered from long-term sickness to a greater extent, and had higher levels of education compared to those with only one valid accelerometer measurement. Mean percentage weight loss at 12-month post-surgery was $47.2 \%(\mathrm{SD}=16.8)$ and prevalence of long-term sickness was also reduced to $27 \%$ at 12 -month post-surgery compared with 59\% pre-surgery.

Table 2 shows participants' levels of PA, LPA, step counts, and ST pre- and 12-month post-surgery. Mean MVPA min/day pre-surgery were $26.4(\mathrm{SD}=21.0)$ and $29.6(\mathrm{SD}=22.4) \mathrm{min} /$ day 12 months after surgery. Participants also increased their time spent in MVPA in 10-min bouts from $7.5(\mathrm{SD}=12.6)$ pre-surgery to $11.6(\mathrm{SD}=14.6)$ $\mathrm{min} /$ day 12 -month post-surgery, although not statistically significant $\mathrm{p}=0.334$. Step counts increased significantly by $1162.8 \mathrm{steps} /$ day $(p=0.014)$ for those with valid data at one measure point, see Table 2. A similar pattern was found for participants with valid accelerometer PA data at both measure points (data not shown). Ten percent of the participants reached the PA recommendation of $\geq 150 \mathrm{~min}$ of MVPA in 10 -min bouts per week pre-surgery and $15 \%$ at 12 -month post-surgery, see Fig. 1.

Table 3 shows the mean SF-36 scores comparing participants engaging in more or less than $150 \mathrm{~min}$ MVPA/week or more or less than 150 min MVPA in 10 min bouts/week. Participants who engaged in more than 150 min MVPA/ week had considerably higher SF-36 scores than those who did not, both pre- and post-surgery. These differences varied greatly for different sub-scores, see Table 3 , and most of the found associations decreased or disappeared when adjusting for confounders, in particular long-term sickness. Looking at the summary scores, PCS showed stronger associations with all levels of PA than MCS after surgery but a strong association between LPA and MCS was found pre-surgery

Table 1 Participants' characteristics and health-related quality of life (HRQoL) before and 12 months after Roux-en-Y gastric bypass surgery (RYGB)

\begin{tabular}{|c|c|c|c|c|}
\hline Variables & Pre-surgery $(n=66)$ & $\begin{array}{l}\text { 12-month post-surgery } \\
(n=62)\end{array}$ & $\begin{array}{l}\text { Pre-surgery with valid } \\
\text { PA measurements at } \\
\text { both time points }(n=39)\end{array}$ & $\begin{array}{l}12 \text {-month post-surgery with } \\
\text { valid PA measurements at both } \\
\text { time points }(n=39)\end{array}$ \\
\hline BMI $\left(\mathrm{kg} / \mathrm{m}^{2}\right)$ & $40.9(5.3)(n=65)$ & $28.1(4.2)(n=59)$ & $40.7(4.3)(n=38)$ & $28.2(3.9)(n=37)$ \\
\hline$\%$ weight loss & & $-47.2 \%(16.8)$ & & $-45.7 \%(15.3)$ \\
\hline Age (years) & $44.5(9.7)$ & $47.5(10.0)$ & $45.8(9.9)$ & $46.8(9.9)$ \\
\hline Education at university level & $21(32 \%)$ & $15(25 \%)$ & $14(36 \%)$ & $13(33 \%)$ \\
\hline Working & $50(76 \%)$ & $51(82 \%)$ & $31(79 \%)$ & $31(79 \%)$ \\
\hline $\begin{array}{l}\text { Suffering from long-term sick- } \\
\text { ness }\end{array}$ & $39(59 \%)$ & $17(27 \%)$ & $24(62 \%)$ & $14(36 \%)$ \\
\hline $\begin{array}{l}\text { Long-term sickness with no } \\
\text { limitations }\end{array}$ & $8(12 \%)$ & $6(10 \%)$ & $8(21 \%)$ & $4(10 \%)$ \\
\hline $\begin{array}{l}\text { Long-term sickness with some } \\
\text { limitations }\end{array}$ & $18(27 \%)$ & $7(11 \%)$ & $8(21 \%)$ & $6(15 \%)$ \\
\hline $\begin{array}{l}\text { Long-term sickness with a high } \\
\text { degree of limitations }\end{array}$ & $13(20 \%)$ & $4(6 \%)$ & $8(21 \%)$ & $4(10 \%)$ \\
\hline \multicolumn{5}{|l|}{ SF-36 subscales } \\
\hline PF, physical functioning & $56.1(24.1)$ & $89.6(15.0)$ & $56.5(25.7)$ & $86.9(17.8)$ \\
\hline $\mathrm{RP}$, physical role functioning & $70.6(30.7)$ & $90.1(18.5)$ & $73.9(29.2)$ & $88.8(19.5)$ \\
\hline BP, bodily pain & $43.8(27.6)$ & $75.4(27.5)$ & $46.0(26.8)$ & $70.5(21.8)$ \\
\hline GH, general health & $50.9(21.2)$ & $79.1(19.5)$ & $50.8(19.4)$ & $77.9(21.2)$ \\
\hline VT, vitality & $37.7(21.3)$ & $64.3(21.1)$ & $38.6(20.1)$ & $65.2(21.9)$ \\
\hline SF, social role functioning & $62.7(28.5)$ & $90.7(19.2)$ & $66.0(27.6)$ & $90.4(20.2)$ \\
\hline $\mathrm{RE}$, emotional role functioning & $76.4(30.3)$ & $91.4(18.0)$ & $75.9(30.6)$ & $90.4(17.4)$ \\
\hline MH, mental health & $62.9(20.9)$ & $81.7(14.1)$ & $64.1(19.3)$ & $81.0(15.5)$ \\
\hline PCS, physical summary score & $41.5(9.2)$ & $53.7(7.6)$ & $42.1(8.5)$ & $52.5(8.6)$ \\
\hline MCS, mental summary score & $45.2(11.2)$ & $53.5(7.8)$ & $45.6(10.1)$ & $53.7(8.1)$ \\
\hline
\end{tabular}

Data presented as mean $(\mathrm{SD})$ or number (percentage)

$B M I$ Body mass index, SF-36 the Short Form (36) Health Survey 
Table 2 Physical activity (PA), sedentary time (ST), and step counts for participants pre-surgery and 12-month postsurgery

\begin{tabular}{|c|c|c|c|}
\hline Variables & Pre-surgery $(n=66)$ & $\begin{array}{l}\text { 12-month post- } \\
\text { surgery }(n=62)\end{array}$ & $\begin{array}{l}\text { Difference between pre- } \\
\text { and post-surgery }(n=39)\end{array}$ \\
\hline Mean wear time $\mathrm{h} /$ day & $14.0(1.2)$ & $14.8(1.4)$ & $\begin{array}{l}0.4(1.6) \\
p=0.089\end{array}$ \\
\hline Mean counts/min & $583.9(229.2)$ & $598.8(180.0)$ & $\begin{array}{l}6.5 \\
p=0.826\end{array}$ \\
\hline MVPA in 10 bouts, min/day & $7.5(12.6)$ & $11.6(14.6)$ & $\begin{array}{l}2.7(16.9) \\
p=0.334\end{array}$ \\
\hline MVPA min/day & $26.4(21.0)$ & $29.6(22.4)$ & $\begin{array}{l}2.1(22.6) \\
p=0.566\end{array}$ \\
\hline LPA min/day & $359.0(88.2)$ & $400.7(78.0)$ & $\begin{array}{l}19.6(93.3) \\
p=0.197\end{array}$ \\
\hline ST min/day & $455.0(99.3)$ & $457.3(100.6)$ & $\begin{array}{l}5.1(94.3) \\
p=0.737\end{array}$ \\
\hline Step counts steps/day & $5971.0(2776.5)$ & $7511.7(2989.0)$ & $\begin{array}{l}1162.8(2829.3) \\
p=0.014\end{array}$ \\
\hline
\end{tabular}

Data presented as mean (SD)

MVPA moderate to vigorous physical activity, LPA light physical activity, ST sedentary time

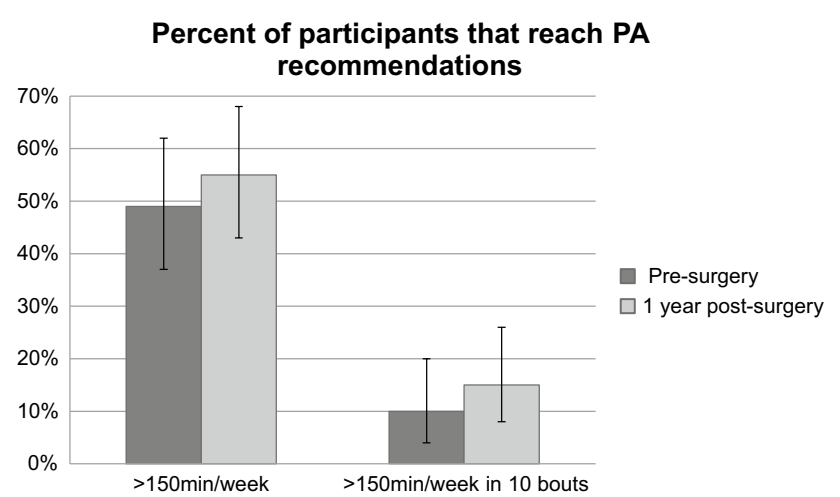

Fig. 1 Percentage of participants meeting physical activity recommendations, pre-, and post-RYGB surgery

with a 2.6-point increase in MCS for each hour increase in LPA, see Table 4 . The results in Table 4 are presented as change in SF-36 score per hour increase in LPA or ST and per 1000 increased steps/day.

\section{Sensitivity analyses}

Sensitivity analyses of mean scores of SF-36 comparing more or less than $150 \mathrm{~min} /$ week of MVPA and more or less than 150 min of MVPA in 10 min bouts/week for participants with valid PA measures at both measure points can be found in Supplementary Table $1(n=39)$. In general, there were slightly weaker associations pre-surgery, and in almost all subscales the associations 12-month post-surgery were stronger, with bodily pain showing very strong association engaging in $\geq 150 \mathrm{~min}$ MVPA/week, in average 29.1 $(p=0.001)$ higher score in bodily pain (i.e., meaning less pain) compared to those who did not; same numbers for engaging in $\geq 150 \mathrm{~min} \mathrm{MVPA} /$ week in $10 \mathrm{~min}$ bouts were 29.5 ( $p=0.008)$. Supplementary Table 2 shows associations between LPA, ST, step counts, and HRQoL for participants with valid PA measures at both measure points; the table shows similar results to the analyses including all participants.

Additionally, we also checked for if changes in physical activity (going from active, meeting PA recommendations, to inactive, not meeting PA recommendations, or the other way around) were associated with changes in HRQoL in these 39 participants. Unfortunately, there were too few participants to see any valid results, see Supplementary Material 3. However, we saw no relevant differences in changes in HRQoL between participants being active before surgery compared to inactive (mean improvement in $\mathrm{MCS}=7.4$ $(\mathrm{SD}=8.3)$ vs. $8.7(\mathrm{SD}=9.7)$ and for $\mathrm{PCS}=9.7(\mathrm{SD}=6.6)$ vs. $11.1(\mathrm{SD}=8.6)$, respectively).

Moreover, we run the same analyses as above including all participants with accelerometer data (without any criteria of wear time). This only added two participants and did not change any of the results substantially.

\section{Discussion}

Meeting the PA recommendations of $\geq 150$ min of MVPA per week in non-bouts and in 10-min bouts was associated with higher HRQoL summary scores as well as in many of the subscales pre- and post-RYGB and associations were found to be stronger post-surgery. Additionally, we also found associations for LPA, ST, and step counts with HRQoL pre- and post-RYGB. However, most of the associations were decreased or diminished when adjusting for relevant confounders, especially when adjusting for living 


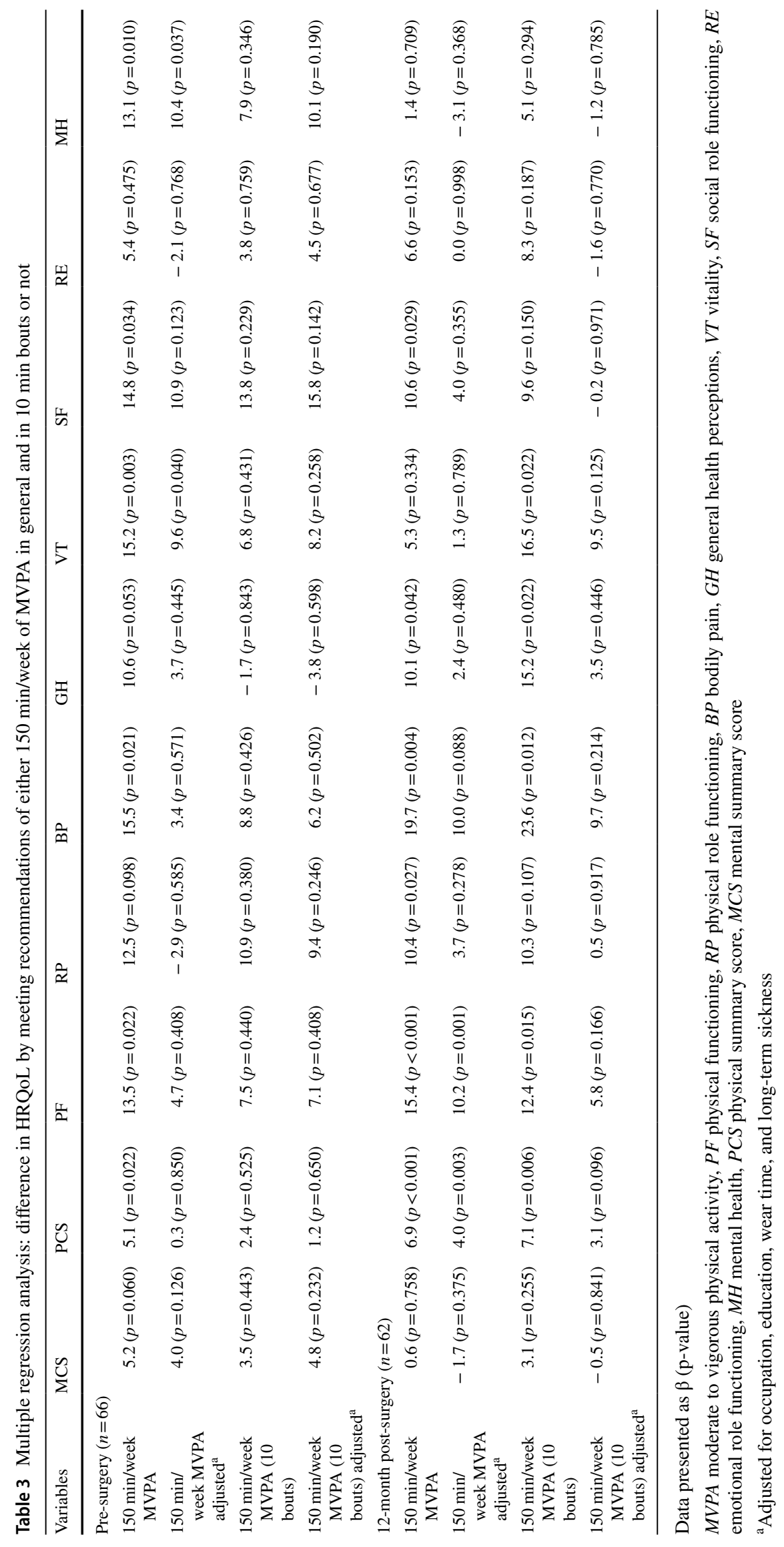




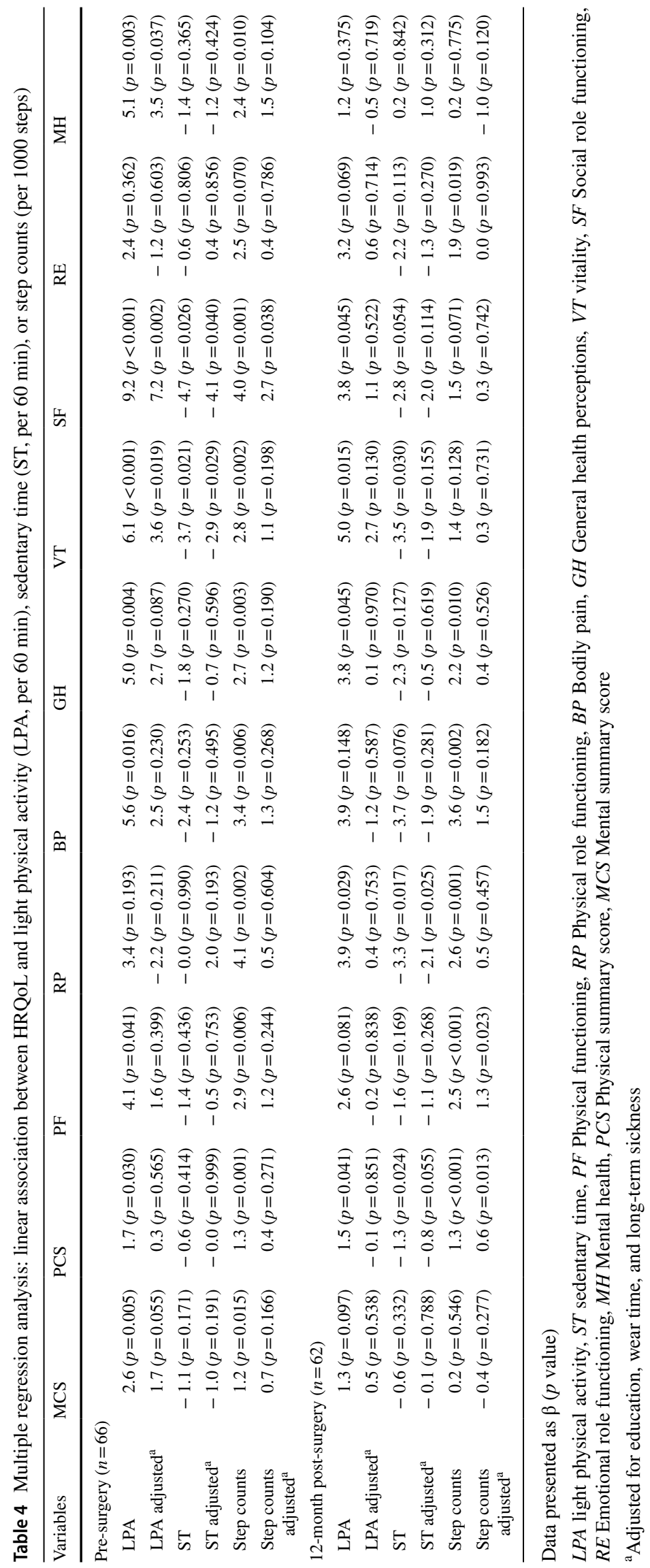


with long-term sickness. In general, participating women improved their HRQoL substantially from pre- to 12-month post-RYGB, especially on subscales connected to the physical part of HRQoL.

Pre-surgery, women's HRQoL scores were considerably lower than among the general population [32]. However, at 12-month post-RYGB, scores were similar, and in some subscales even higher, than among the general population [32]. All intensity levels of PA were higher 12-month postsurgery compared to pre-surgery, although not statistically significant, except for step counts. Great improvements in HRQoL after bariatric surgery, in line with our results, have been seen in several previous studies [12, 13, 23], with a peak improvement at 12-month post-surgery [33]. Contrary to previous studies on objectively measured PA after bariatric surgery $[34,35]$, participants in the current study increased both MVPA and LPA at 12-month post-surgery. However, this increase was quite small and statistically nonsignificant. Pre-surgery, approximately 50\% meet the recommended levels of $\geq 150$ min of MVPA per week, compared with 55\% 12-month post-surgery. On the other hand, only $10 \%$ pre- and $15 \% 12$-month post-surgery met the recommended levels of $\geq 150$ min of MVPA per week performed in 10-min bouts, as stated in the PA recommendations [30]. These proportions of participants meeting the PA recommendations are similar to those reported in a previous study with objectively measured PA in women undergoing RYGB [36], with the exception of fewer women meeting $150 \mathrm{~min}$ MVPA per week pre-surgery in the current study.

As stated before, the general recommendation for PA for the age group 17-64 is at least 150 min of weekly MVPA performed in bouts of at least $10 \mathrm{~min}$ [30]. We chose to additionally look at 150 min of MVPA per week in non-bouts, since few participants met the PA recommendation and due to the fact that recently published data show that MVPA, independent of how it is accumulated throughout the day, is associated with numerous health benefits [31]. Although meeting the recommended levels of PA is an appropriate long-term goal for most RYGB patients, this may be too challenging for many patients. Thus, setting realistic, achievable, assessable short-term goals (e.g., 150 min MVPA per week in non-bouts), and gradually increasing the amount and intensity of PA over time may be a conceivable strategy [37].

In general, the associations between meeting the PA recommendations of $\geq 150$ min of MVPA per week and higher HRQoL tended to be stronger at 12-month post-RYGB compared to pre-surgery, both for general HRQoL scores and for the different subscales. Both levels of MVPA and scores for the subscales connected to the physical part of HRQoL increased 12-month post-surgery. This could be due to the importance of physical health among severely obese individuals, where for example, bodily pain was scored much higher (high scores indicate less pain) for participants that were able to and were active $\geq 150$ min of MVPA per week. When taking into account long-term sickness, the association between meeting the PA recommendations and HRQoL became weaker, supporting this theory. For the subgroup of 39 women who had accelerometer data at both measurement points, the association between meeting PA recommendations and HRQoL was somewhat stronger. They also had a somewhat higher prevalence of long-term sickness, which is in line with the theory above. However, the mean levels of PA in this patient group did not increase post-surgery. Thus, the slightly increased association might also be a function of weight loss, although this is only speculative. The strong associations seen between meeting the $150 \mathrm{~min}$ of MVPA per week recommendations and SF-36 were also seen for LPA, step counts, and ST, although difficult to compare since they were analyzed as continuous outcomes. However, increasing LPA with $1 \mathrm{~h}$ or increasing with 1000 steps per day might be preferred for some patients. Thus, an increase in both MVPA, LPA, and step counts could be recommended for this patient group.

A previous PA intervention study with objectively measured PA, in 75 pre- bariatric surgery patients, showed that patients who reached larger increases in MVPA also displayed greater improvements in HRQoL, especially for the physical health subscales (physical function, bodily pain, general health, and overall physical health) regardless of age, degree of obesity, and initial baseline levels of MVPA and HRQoL [38]. A similar study also found higher levels of some parts of HRQoL (general health and role physical) after a 24-week physical training intervention compared to a control group [23]. Another study assessing associations between PA and HRQoL before and after bariatric surgery found that patients who were inactive before and became active after surgery and those who were active before and after surgery reported greater improvements in HRQoL, especially in the physical health scores, compared with patients who were active before and became inactive after surgery and those who were inactive both before and after surgery [39]. However, those findings have limited comparability with results from the current study since the study used self-reported data on PA which has shown large discrepancies compared to objective measures of PA, which seem to be even greater after, compared to before, bariatric surgery [24]. Future research should consider intervention settings where levels of PA are manipulated at pre- and postRYGB (and measured objectively) to study potential effects on HRQoL.

\section{Limitations}

The results of the current study should be interpreted in light of its limitations. The inclusion criteria used to assess participants' eligibility to enroll in the study purposefully 
excluded those with $<10 \mathrm{~h}$ of daily accelerometer wear time data for $<3$ days; this was not a problem since including those two participants with less accelerometer data than $10 \mathrm{~h}$ at least 3 days did not change the results. Additionally, participants in these analyses arise from RYGB patients that wanted to participate in a RCT improving HRQoL and PA after surgery and may therefore have influenced the findings of the study. We cannot tell whether increased PA results in better HRQoL or if better HRQoL makes people more active, since the analyses are cross-sectional and we lacked statistical power to do longitudinal analyses. However, a previous study on obese individuals waiting for RYGB surgery showed greater improvements in HRQoL in an intervention group who increased their PA compared to a control group who did not increase their levels of PA [22]. Secondly, we adjusted for long-term sickness which could act as a moderator, rather than a confounder. However, we still believe that long-term sickness can affect PA levels and HRQoL and therefore can be seen as a confounder. Thirdly, the majority of participants only had valid accelerometer data at one measure point and there could be systematic differences in descriptive and outcome variables between these participants and those who had valid PA data at both measure points. However, we conducted sensitivity analyses using this subgroup and there were indeed differences both in the baseline characteristics and the association between PA, step counts, ST, and SF-36 scores, although this could be due to unstable results because of the small sample size in the sensitivity analyses $(n=39)$. At baseline, long-term sickness was more prevalent; education levels were higher; and HRQoL scores were slightly higher among those with both valid PA measurement points. Fourth, one potential limitation from using objectively accelerometer measured PA and $\mathrm{ST}$ is that the method is not able to specify specific domains or types of PA and ST (e.g., occupational/transportation PA/ ST or sitting vs. standing still). A final potential limitation related to the lack of consensus on how to define intensities of PA via accelerometer data intensity cut-offs. This limits the comparability with other studies having used different cut-offs to define PA intensities.

\section{Strengths}

This study also shows several strengths. The main strength is the objectively measured PA and ST in 66 obese individuals pre-RYGB and 62 individuals at 12-month follow-up. Another strength is that we were able to examine 12-month post-surgery data, i.e., a period when most RYGB patients have recovered well enough from the surgery to be active again. However, a longer follow-up than 12 month would be of interest since it is known that HRQoL decreases again 1-2 years post-surgery [33]. Further strengths include the objective height and weight measures which were made in a standardized manner, in a hospital setting, both pre- and post-RYGB, which ensures the accuracy of weight loss data.

\section{Conclusions}

The current study found a strong association between meeting guideline PA levels of at least 150 min of MVPA per week and higher HRQoL both pre- and 12-month postRYGB surgery. Similar results were found for LPA, ST, and step counts both pre- and post-surgery. There were overall higher scores on subscales measuring the physical part of HRQoL, compared to mental parts (both with strongest association for bodily pain and vitality and no relevant associations for the subscale measuring emotional role functioning). These results raise a hypothesis of a possible effect of PA, especially meeting the recommendations of $\geq 150 \mathrm{~min}$ MVPA per week either in non-bouts or in 10-min bouts, for obese individuals in general but also after RYGB surgery to improve and keep a higher quality of life.

Acknowledgements We would like to thank the staff involved in this study from the five hospitals, Danderyds Hospital, Ersta Hospital, Uppsala University Hospital, Örebro University Hospital, and St. Görans Hospital, for their help in recruiting study participants and to the study participants that participated in the data collection.

Funding This study was funded by a grant to Daniel Berglind from the Swedish Scientific Council (Vetenskapsrådet) with Grant No. 201502621, Erling-Persson Family Foundation, and the Research School of Caring Sciences, Karolinska Institutet.

\section{Compliance with ethical standards}

Conflict of interest The authors declare that they have no conflict of interest.

Ethical approval All procedures performed in studies involving human participants were in accordance with the ethical standards of the institutional and/or national research committee and with the 1964 Helsinki Declaration and its later amendments or comparable ethical standards. The study was approved by the regional ethics committee of Stockholm (Dnr:2013/1847-31/2). The trial has also been registered at http://www. isrctn.org with identification number ISRCTN16417174.

Informed consent Informed consent was obtained from all individual participants included in the study.

Open Access This article is distributed under the terms of the Creative Commons Attribution 4.0 International License (http://creativeco mmons.org/licenses/by/4.0/), which permits unrestricted use, distribution, and reproduction in any medium, provided you give appropriate credit to the original author(s) and the source, provide a link to the Creative Commons license, and indicate if changes were made. 


\section{References}

1. Warburton, D. E., Nicol, C. W., \& Bredin, S. S. (2006). Health benefits of physical activity: The evidence. CMAJ, 174(6), 801-809.

2. Arem, H., et al. (2015). Leisure time physical activity and mortality: A detailed pooled analysis of the dose-response relationship. JAMA Internal Medicine, 175(6), 959-967.

3. Bize, R., Johnson, J. A., \& Plotnikoff, R. C. (2007). Physical activity level and health-related quality of life in the general adult population: A systematic review. Preventive Medicine, 45(6), 401-415.

4. Kolt, G. S., et al. (2017). Associations between quality of life and duration and frequency of physical activity and sedentary behaviour: Baseline findings from the WALK 2.0 randomised controlled trial. PLoS ONE, 12(6), e0180072.

5. Hart, P. D., Benavidez, G., \& Erickson, J. (2017). Meeting recommended levels of physical activity in relation to preventive health behavior and health status among adults. Journal of Preventive Medicine \& Public Health, 50(1), 10-17.

6. Romero, M., Vivas-Consuelo, D., \& Alvis-Guzman, N. (2013). Is health related quality of life (HRQoL) a valid indicator for health systems evaluation? Springerplus, 2, 664.

7. Loprinzi, P. D. (2015). Joint associations of objectively-measured sedentary behavior and physical activity with health-related quality of life. Preventive Medicine Reports, 2, 959-961.

8. Rabel, M., et al. (2017). The longitudinal association between change in physical activity, weight, and health-related quality of life: Results from the population-based KORA S4/F4/FF4 cohort study. PLoS ONE, 12(9), e 0185205.

9. Jepsen, R., et al. (2013). Associations between physical activity and quality of life outcomes in adults with severe obesity: A crosssectional study prior to the beginning of a lifestyle intervention. Health and Quality of Life Outcomes, 11, 187.

10. Kroes, M., et al. (2016). Impact of weight change on quality of life in adults with overweight/obesity in the United States: A systematic review. Current Medical Research and Opinion, 32(3), 485-508.

11. Kolotkin, R. L., Meter, K., Williams, G. R. (2001). Quality of life and obesity. Obesity Reviews, 2(4), 219-229.

12. Monpellier, V. M., et al. (2017). Improvement of health-related quality of life after Roux-en-Y gastric bypass related to weight loss. Obesity Surgery, 27(5), 1168-1173.

13. Karlsen, T. I., et al. (2013). Health related quality of life after gastric bypass or intensive lifestyle intervention: A controlled clinical study. Health and Quality of Life Outcomes, 11, 17.

14. O'Brien, P. E., et al. (2006). Treatment of mild to moderate obesity with laparoscopic adjustable gastric banding or an intensive medical program: A randomized trial. Ann Intern Med, 144(9), 625-633.

15. SOReg, S.O.S.R., Årsrapport SOReg 2016. Del 1-operationsstatistik och tidiga komplikationer 2017. Retrieved from http://www. ucr.uu.se/soreg/arsrapporter.

16. Driscoll, S., et al. (2016). Long-term health-related quality of life in bariatric surgery patients: A systematic review and metaanalysis. Obesity (Silver Spring), 24(1), 60-70.

17. SOReg, S. O. S. R. Årsrapport SOReg 2016. Del 3. Livskvalitet, Mortalitet, Datakvalitet, Forskning 2017. Retrieved from http:// www.ucr.uu.se/soreg/arsrapporter.

18. Bartsch, M., et al. (2016). Physical activity, decision-making abilities, and eating disturbances in pre- and postbariatric surgery patients. Obesity Surgery, 26(12), 2913-2922.

19. Crisp, A. H., et al. (2017). Changes in physical activities and body composition after roux-Y gastric bypass surgery. Obesity Surgery, 28(6), 1665-1671.
20. Herring, L. Y., et al. (2016). Changes in physical activity behaviour and physical function after bariatric surgery: A systematic review and meta-analysis. Obesity Reviews, 17(3), 250-261.

21. Berglind, D., et al. (2016). Accelerometer-measured versus selfreported physical activity levels and sedentary behavior in women before and 9 months after roux-en-Y gastric bypass. Obesity Surgery, 26(7), 1463-1470.

22. Bond, D. S., et al. (2015). Exercise improves quality of life in bariatric surgery candidates: Results from the bari-active trial. Obesity (Silver Spring), 23(3), 536-542.

23. Stolberg, C. R., et al. (2018) Physical training following gastric bypass: effects on physical activity and quality of life - a randomized controlled trial. Quality of Life Research, 27(12), 3113-3122.

24. Berglind, D., et al. (2015). Accelerometer-measured versus selfreported physical activity levels and sedentary behavior in women before and 9 months after roux-en-Y gastric bypass. Obesity Surgery, 26(7), 1463-1470.

25. Sellberg, F., et al. (2018). A dissonance-based intervention for women post roux-en-Y gastric bypass surgery aiming at improving quality of life and physical activity 24 months after surgery: Study protocol for a randomized controlled trial. BMC Surgery, 18(1), 25.

26. Obidoa, C. A., et al. (2010). How does the SF-36 perform in healthy populations? A structured review of longitudinal studies. Journal of Social, Behavioral, and Health Sciences, 4(1), 30-48.

27. Choi, L., et al. (2011). Validation of accelerometer wear and nonwear time classification algorithm. Medicine \& Science in Sports \& Exercise, 43(2), 357-364.

28. Troiano, R. P., et al. (2008). Physical activity in the United States measured by accelerometer. Medicine \& Science in Sports \& Exercise, 40(1), 181-188.

29. Santos-Lozano, A., et al. (2013). Actigraph GT3X: Validation and determination of physical activity intensity cut points. International Journal of Sports Medicine, 34(11), 975-982.

30. WHO. (2010). Global recommendations on physical activity for health. Geneva: WHO Press.

31. Saint-Maurice, P. F., et al. (2018). Moderate-to-vigorous physical activity and all-cause mortality: Do bouts matter? Journal of the American Heart Association, 7(6), e007678

32. Sullivan, M., Karlsson, J., \& Ware, J. E. Jr. (1995). The Swedish SF-36 Health Survey-I. Evaluation of data quality, scaling assumptions, reliability and construct validity across general populations in Sweden. Social Science \& Medicine, 41(10), 1349-1358.

33. Karlsson, J., et al. (2007). Ten-year trends in health-related quality of life after surgical and conventional treatment for severe obesity: The SOS intervention study. International Journal of Obesity (London), 31(8), 1248-1261.

34. Berglind, D., et al. (2015). Longitudinal assessment of physical activity in women undergoing Roux-en-Y gastric bypass. Obesity Surgery, 25(1), 119-125.

35. Bond, D. S., et al. (2010). Pre- to postoperative physical activity changes in bariatric surgery patients: Self report vs. objective measures. Obesity (Silver Spring), 18(12), 2395-2397.

36. Sellberg, F., et al. (2017). Four years' follow-up changes of physical activity and sedentary time in women undergoing roux-en-Y gastric bypass surgery and appurtenant children. BMC Surgery, 17(1), 133.

37. King, W. C., \& Bond, D. S. (2013). The importance of preoperative and postoperative physical activity counseling in bariatric surgery. Exercise and Sport Sciences Reviews, 41(1), 26-35.

38. Bond, D. S., et al. (2015). Exercise improves quality of life in bariatric surgery candidates: Results from the bari-active trial. Obesity, 23(3), 536-542. 
39. Bond, D., et al. (2009). Becoming physically active after bariatric surgery is associated with improved weight loss and health-related quality of life. Obesity (Silver Spring), 17(1), 78-83.

Publisher's Note Springer Nature remains neutral with regard to jurisdictional claims in published maps and institutional affiliations. 